ПЕРСИСТЕНЦИЯ ВТОРИЧНОГО ГИПЕРПАРАТИРЕОЗА ПОСЛЕ РАНЕЕ ПРОВЕДЕННОЙ ПАРАТИРЕОИДЭКТОМИИ У ПАЦИЕНТКИ С ХРОНИЧЕСКОЙ БОЛЕЗНЬЮ ПОЧЕК: КЛИНИЧЕСКИЙ СЛУЧАЙ

(с) Е.А. Ильичева ${ }^{1}$, Г.А. Берсенев ${ }^{1 *}$, Д.А. Булгатов ${ }^{1}$, В.Н. Махутов ${ }^{2}$

'Федеральное государственное бюджетное научное учреждение «Иркутский научный центр хирургии и травматологии», г. Иркутск, Россия

2Государственное бюджетное учреждение здравоохранения «Иркутская ордена "Знак почета" областная клиническая больница», г. Иркутск, Россия

Представлено клиническое наблюдение хирургического лечения персистенции вторичного гиперпаратиреоза (ВГПТ) у пациентки с хронической болезнью почек после ранее проведенной паратиреоидэктомии (ПтЭ). Программный гемодиализ начат в 2014 г., а в 2016 г. диагностирован ВГПТ. В ноябре 2018 г. в одной из клиник были выставлены показания к оперативному лечению. По данным сцинтиграфии наблюдалось повышение функциональной активности всех четырех околощитовидных желез (ОЩЖ), по данным УзИ установлена локализация правой нижней, левой верхней и нижней ОЩЖ. Было проведено удаление 3 ОЩЖ без ревизии рогов тимуса (11.2018), при этом в послеоперационном периоде адекватного снижения паратгормона не наблюдалось. После дообследования по данным ультразвукового исследования, сцинтиграфии и мультиспиральной компьютерной томографии шеи с внутривенным усилением было установлено, что обе нижние ОЩЖ располагаются в верхних рогах тимуса. В июле 2020 г. выполнено повторное оперативное вмешательство в объеме правой нижней и левой нижней ПТЭ с положительной интраоперационной пробой. Ремиссия ВГПТ была достигнута. В настоящее время консенсуса в отношении объема оперативного вмешательства при ВГПт не достигнуто, поскольку не обнаружено статистически значимых различий в частоте персистенции/рецидива ВГпт. Необходима полная оценка результатов предоперационных методов визуализации при планировании оперативного вмешательства. Интраоперационный мониторинг ПТГ является эффективной методикой для оценки радикальности оперативного лечения.

КЛЮЧЕВЫЕ СЛОВА: вторичный гиперпаратиреоз; хроническая болезнь почек; персистенция гиперпаратиреоза; хирургическое лечение; клинический случай.

\title{
PERSISTENCE OF SECONDARY HYPERPARATHYROIDISM AFTER PARATHYROIDECTOMY IN A PATIENT WITH CHRONIC KIDNEY DISEASE: A CASE REPORT
}

(c) Elena A. Ilyicheva' ${ }^{1}$, Gleb A. Bersenev ${ }^{1 *}$, Dmitry A. Bulgatov ${ }^{1}$, Valeriy N. Makhutov²

\author{
${ }^{1}$ Irkutsk Scientific Centre of Surgery and Traumatology, Irkutsk, Russian Federation \\ ${ }^{2}$ Irkutsk Regional Clinical Hospital, Irkutsk, Russian Federation
}

This is a clinical case of surgical treatment of persistence of secondary hyperparathyroidism (SHPT) in a patient with chronic kidney disease after previous parathyroidectomy. Programmed hemodialysis started in 2014, and in 2016 SHPT was diagnosed. In November 2018, indications for surgical treatment were presented in one of the clinics. According to scintigraphy, an increase in the functional activity of all four parathyroid glands (PTG) was observed, according to ultrasound data, the localization of the right lower, left upper and lower PTG was established. Removal of 3x PTG was performed without revision of the thymus horns (11.2018), while in the postoperative period, an adequate decrease in parathyroid hormone was not observed. After additional examination, according to ultrasound, scintigraphy and computer tomography of the neck with intravenous enhancement, it was found that both lower PTGs are located in the upper horns of the thymus. In July 2020, a repeated surgical intervention was performed in the volume of the right lower and left lower parathyroidectomy with a positive intraoperative test. Remission of SHPT was achieved. At present, no consensus has been reached on the extent of surgery for SHPT, since no statistically significant differences were found in the incidence of persistence / recurrence of SHPT. A complete assessment of the results of preoperative imaging techniques is required when planning surgery. Intraoperative monitoring of parathyroid hormone is an effective technique for assessing the radicality of surgical treatment.

KEYWORDS: secondary hyperparathyroidism; chronic kidney disease; persistence of hyperparathyroidism; surgical treatment; clinical case. 


\section{АКТУАЛЬНОСТЬ}

Заболевания околощитовидных желез (ОЩЖ) находятся на третьем месте по распространенности среди всей эндокринологической патологии [1]. В нашей стране вторичный гиперпаратиреоз (ВГПТ) диагностирован у 27,7\% пациентов, получающих заместительную почечную терапию (ЗПТ) диализом [2]. У этих пациентов целевой уровень паратиреоидного гормона (ПТГ) составляет 150-300 пг/мл, а допустимые значения - от 130 до 600 пг/мл [3]. У 30,9\% пациентов, которые получают ЗПТ в России, наблюдается уровень ПТГ более 600 пг/мл [4].

Успешное оперативное лечение ВГПт позволяет улучшить качество жизни пациентов, снижает риск сердечно-сосудистых осложнений и связанной с ними летальности [5]. Потенциальными осложнениями данного метода лечения являются гипопаратиреоз, персистенция и рецидив ВГПТ [3].

Сохраняется дискуссия в отношении цели и объема оперативного вмешательства. Не определена конечная точка лечения данных пациентов: достижение целевых значений ПТГ или послеоперационного гипопаратиреоза [6]. Нет единого понимания необходимого объема операции, а также критериев оценки результатов лечения $[7,8]$.

В настоящее время применяются следующие объемы оперативных вмешательств при ВГПт: субтотальная паратиреоидэктомия (ПТЭ), тотальная ПТЭ (с или без аутотрансплантации (АТ) ткани ОЩЖ; с или без удаления верхних рогов тимуса), расширенные диссекции шеи и верхнего средостения [3], а также селективные ПТЭ (удаление только аденоматозно измененных ОЩЖ) [9].

Согласно рекомендациям KDIGO, оптимальным объемом оперативного вмешательства считается субтотальная или тотальная ПТЭ с АТ ткани ОЩЖ [8]. При этом субтотальная ПТЭ связана с более высоким риском рецидива [10]. Общая частота персистенции/рецидивов ВГПТ после первичного хирургического лечения составляет от 8 до 38\% [11-16]. Персистенция после тотальной ПТЭ с АТ наблюдается в 0,4-16\% случаев [11-14], тотальной ПТЭ без АТ - 0,5-6,2\% [11,14], субтотальной ПТЭ - 3,1-22\% [11,13,15-16] и селективной ПТЭ - 4,1$25 \%[11,16]$.

Приводим клиническое наблюдение хирургического лечения пациентки с персистенцией ВГПТ на фоне хронической болезни почек (ХБП) после ранее проведенной ПТЭ.

\section{ОПИСАНИЕ НАБЛЮДЕНИЯ}

Пациентка 33 лет в течение 20 лет наблюдалась у нефролога по месту жительства с врожденной аномалией развития мочевыделительной системы, которая осложнилась двусторонним пузырно-мочеточниково-лоханочным рефлюксом и рефлюкс-нефропатией. С 2007 г. диагностирован рост уремических показателей. Спустя 7 лет установлена терминальная стадия ХБП, и по экстренным показаниям начата ЗПТ в виде сеансов гемодиализа. Через год выполнена двусторонняя нефрэктомия в рамках подготовки к аллотрансплантации почки. С 2016 г. регистрируется повышенный уровень ПтГ более 1000 пг/мл. В течение всего периода наблюдения, несмотря на соблюдение гипофосфатной диеты, прием фосфатбиндеров, попытки интенсификации гемодиализа, отмечалось значительное повышение уровней фосфора и ПТГ. В редкие периоды нормализации уровня фосфора крови применялись активные формы витамина D. B это же время у пациентки появились жалобы на общую слабость, боль в костях и суставах. Ультразвуковое исследование (УЗИ) шеи (05.2016) показало наличие гипоэхогенных образований у обоих нижних полюсов щитовидной железы (ЩЖ) размерами $0,7 \times 0,8 \times 0,4$ см справа и 0,6×0,5×0,4 см слева. По данным сцинтиграфии (07.2017) выявлено повышение функциональной активности всех четырех ОЩЖ (рис. 1).

Несмотря на длительно получаемую комплексную терапию цинакальцетом 90 мг в сутки, парикальцитолом 15 мкг 3 раза в неделю, отмечалась отрицательная динамика показателей фосфорно-кальциевого обмена. По данным УЗИ шеи (20.11.2018): правая верхняя ОЩЖ не визуализируется. Левая верхняя ОЩЖ располагается за левой долей ЩЖ на границе ее средней и нижней трети в виде гипоэхогенного образования размерами 1,5×1,0×0,6см. Левая нижняя ОЩЖ располагается на 1 см ниже нижнего полюса левой доли Щж размерами $0,9 \times 0,8 \times 0,6 \mathrm{~cm}$. Правая нижняя ОЩЖ - за нижним полюсом правой доли ЩЖ размерами $2,1 \times 1,5 \times 0,6$ см. В биохимическом анализе крови (17.03.2019) уровень общего кальция - 2,34 ммоль/л (2,1-2,6 ммоль/л), ионизированного кальция - 1,23 ммоль/л (1,15-1,27 ммоль/л), фосфора — 1,55 ммоль/л (0,81-1,45 ммоль/л), ПТГ 2810,14 пг/мл $(15,0-68,3$ пг/мл). В одной из клиник 02.04.2019 пациентке проведено оперативное вмешательство в объеме цервикотомии, удаления гиперплазированных левой верхней, нижней и правой нижней ОЩЖ с применением нейромониторного контроля возврат-

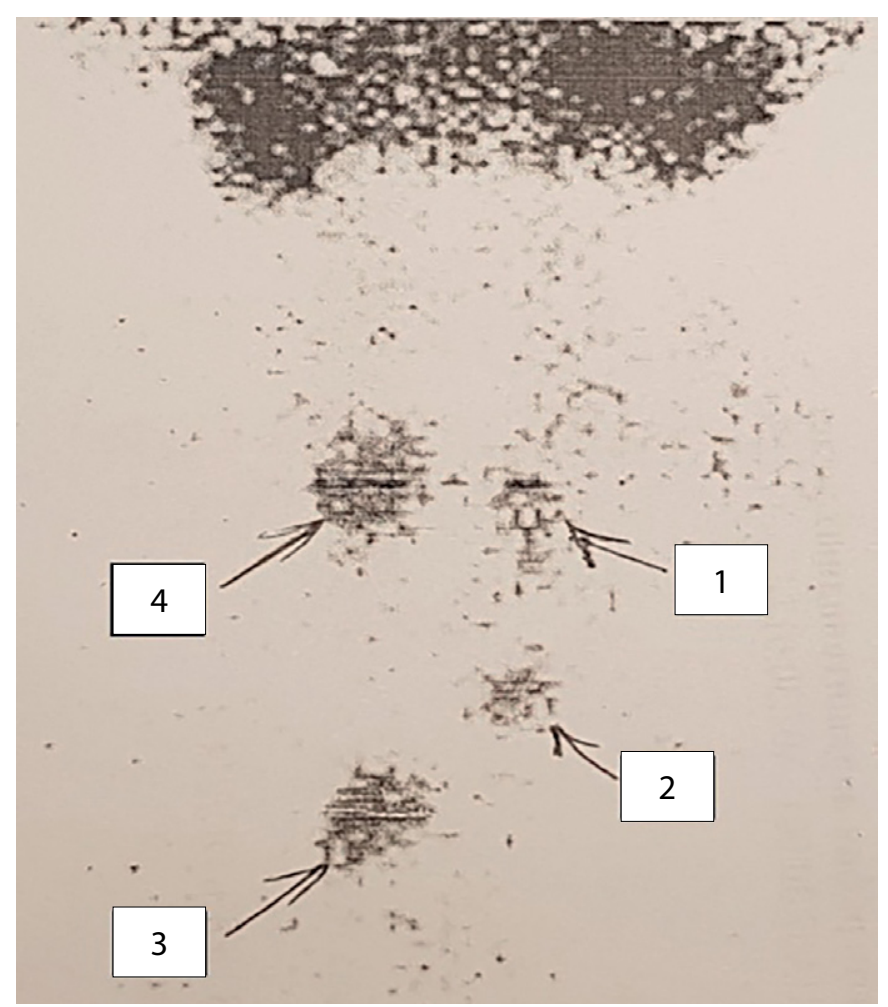

Рисунок 1. Сцинтиграмма шеи в паратиреоидную фазу.

Стрелками указаны анатомические структуры: 1 - левая верхняя ОЩЖ; 2 - левая нижняя ОЩЖ; 3 - правая нижняя ОЩЖ; 4 - правая верхняя ОЩЖ. 


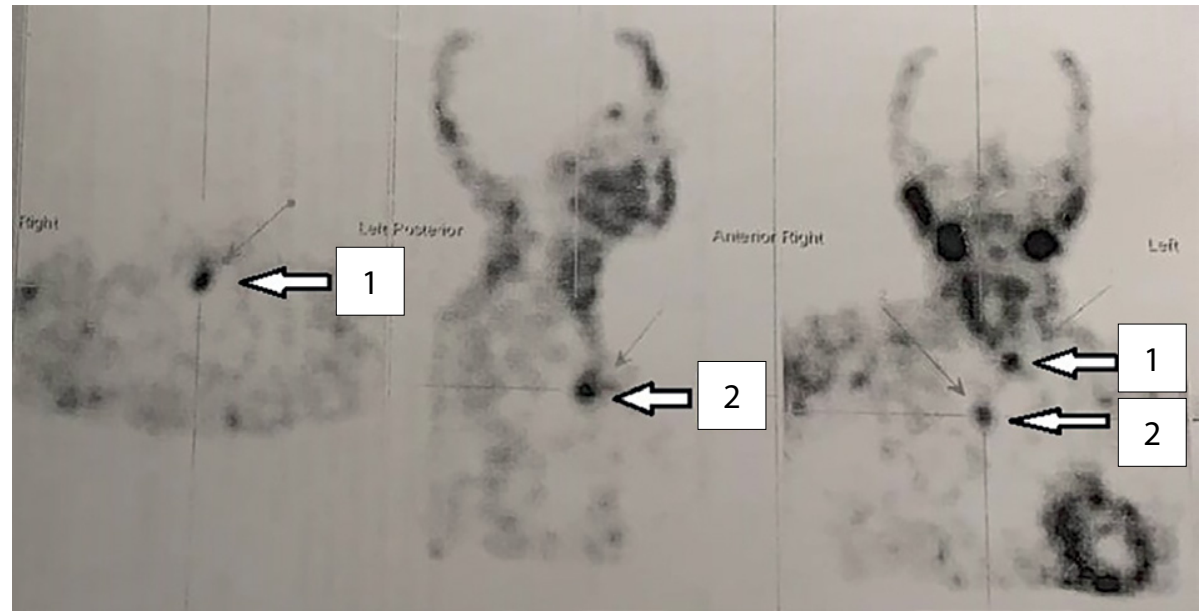

Рисунок 2. Сцинтиграмма шеи в паратиреоидную фазу.

Стрелками указаны анатомические структуры: 1 - левая нижняя ОЩЖ; 2 - правая нижняя ОЩЖ.

но-гортанных нервов (ВГН), интраоперационного мониторинга ПТГ без ревизии рогов тимуса. Правая нижняя ОЩЖ интраоперационно не обнаружена. Данные о динамике интраоперационного мониторинга ПТГ в протоколе операции отсутствуют. В послеоперационном периоде наблюдалось незначительное снижение суточного ПТГ (03.04.2019) - 1976 пг/мл по сравнению с предоперационным уровнем (02.04.2019) - 2596 пг/мл. Учитывая отсутствие адекватного снижения ПтГ в послеоперационном периоде, пациентке было рекомендовано дообследование (УЗИ, сцинтиграфия, мультиспиральная компьютерная томография (МСКТ) органов шеи с внутривенным усилением) через 3 мес. 22.07.2020 пациентка поступила в отделение торакальной хирургии ГБУЗ ИОКБ для повторного оперативного лечения.

\section{Результаты физикального, лабораторного}

и инструментального исследований

Пациентка пониженного питания - рост 142 см, вес 35,8 кг, ИМТ 17,8 кг/м². Кожа бледно-розовой окраски, подкожная жировая клетчатка развита недостаточно. При осмотре области шеи - ЩЖ не увеличена, дополнительные образования не пальпируются. В легких дыхание везикулярное, хрипов нет. Тоны сердца приглушены, ритмичные. Частота сердечных сокращений и пульс 80 в минуту. Артериальное давление на обеих плечевых артериях 136/80 мм рт.ст.

По данным лабораторного обследования (22.07.2020): уровень альбумин-скорректированного кальция 2,49 ммоль/л (2,1-2,6 ммоль/л), ионизированного кальция - 1,24 ммоль/л (1,15-1,27 ммоль/л), фосфора 1,91 ммоль/л (0,81-1,45 ммоль/л), ПТГ - 3179,8 пг/мл (15,0 - 68,3 пг/мл). Минимальный Z-показатель по данным остеоденситометрии (28.05.2020) был -3,8 в поясничном отделе позвоночника.

УЗИ шеи (01.06.2020) показало: левая нижняя ОЩЖ размерами 1,1×0,6×0,7 см располагается ближе к левому грудино-ключичному сочленению. Правая нижняя ОЩЖ размерами 0,8×0,6×0,4 см располагается у правого грудино-ключичного сочленения. На сцинтиграфии с использованием радиофармпрепарата99mТс-технетрил (22.06.2020) выявлено повышение функциональной активности правой и левой нижних ОЩЖ, которые расположены загрудинно (рис. 2). МСКТ шеи с внутривенным усилением(24.07.2020) показала наличие в верхних отделах переднего средостения округлых образований: справа размерами $1,1 \times 0,7 \times 0,6 \mathrm{~cm}$, нативной плотностью 66 ед.Н.; слева размерами $0,8 \times 0,6 \times 0,4$ см, нативной плотностью 36 ед.Н. Образования накапливают контраст: справа до 217 ед.Н. и слева до 84 ед.Н. (рис. 3).

Учитывая персистенцию ВГПТ с высокими показателями ПТГ (>3000 пг/мл) и фосфора крови (>1,9 ммоль/л), показано повторное оперативное вмешательство. Согласно проведенному предоперационному обследованию, объем операции - цервикотомия, правая нижняя и левая нижняя паратиреоидэктомия с интраоперационным мониторингом ПТГ.

Операция состоялась 27.07.2020. Выполнены цервикотомия и доступ к нижним долям ЩЖ. Учитывая выраженный рубцовый процесс, ВГН не выделялись. Левая нижняя ОЩЖ найдена в тиротимической связке, размерами 1,0×1,0×0,8 см, темно-коричневого цвета. Правая нижняя ОЩЖ расположена в верхнем роге тимуса справа, размерами 2,0×1,0×1,0 cм, темно-коричневого цвета (рис. 4а). Произведена мобилизация и удаление левой и правой нижних ОЩЖ в блоке с верхними рогами тимуса (рис. 46). Динамика уровня интраоперационного мониторинга ПТГ была такова: до кожного разреза 2434,2 пг/мл; на момент мобилизации обоих ОЩЖ 925 пг/мл; спустя 10 минут после удаления - 430 пг/мл. Интраоперационная проба - положительная.

Согласно проведенному гистологическому исследованию, правая и левая нижняя ОЩЖ представлены диффузной гиперплазией из активных главных клеток, без собственной капсулы и ободка неизмененной ткани (рис. 5).

В послеоперационном периоде проведена ларингоскопия - установлена симметричная подвижность голосовых складок. На 1-е сутки после операции уровень ПТГ составил 0,7 пг/мл, уровень альбумин-скорректированного кальция - 1,99 ммоль/л. Была назначена заместительная терапия в объеме таблеток карбоната кальция 6 г в сутки и альфакальцидола 4 мкг в сутки. Гипокальциемия купирована. Пациентка выписана на 7-е сутки после операции под наблюдение амбулаторного хирурга, эндокринолога и нефролога.

Осмотрена через 6 мес. после операции. Пациентка жалоб не предъявляет. Уровень ПТГ - 14 пг/мл, уровень 
альбумин-скорректированного кальция - 2,15 ммоль/л. Доза заместительной терапии: таблетки карбоната кальция - 9 г в сутки, альфакальцидола - 4 мкг в сутки.

\section{ОБСУЖДЕНИЕ}

Частота персистенции и рецидива ВГПТ после хирургического лечения связана с качеством проводимой двусторонней ревизии шеи, которая направлена на обнаружение аномально расположенных и добавочных ОЩЖ $[17,18]$. Поэтому предоперационная визуализация всех ОЩЖ имеет высокое значение для успеха оперативного вмешательства при ВГПт.

С целью предоперационной оценки локализации патологически измененных ОЩЖ применяются следующие методы визуализации:УЗИ, сцинтиграфия, МСКТ, однофотонная эмиссионная компьютерная томография (ОФЭКТ/ КТ) и их сочетание. Установлено, что диагностическая точность (ДТ) данных методов визуализации для обнаружения типично расположенных ОЩЖ составила: УЗИ $57,3 \%$, сцинтиграфия - 42,7\%, МСКТ - 60\%. Сочетание
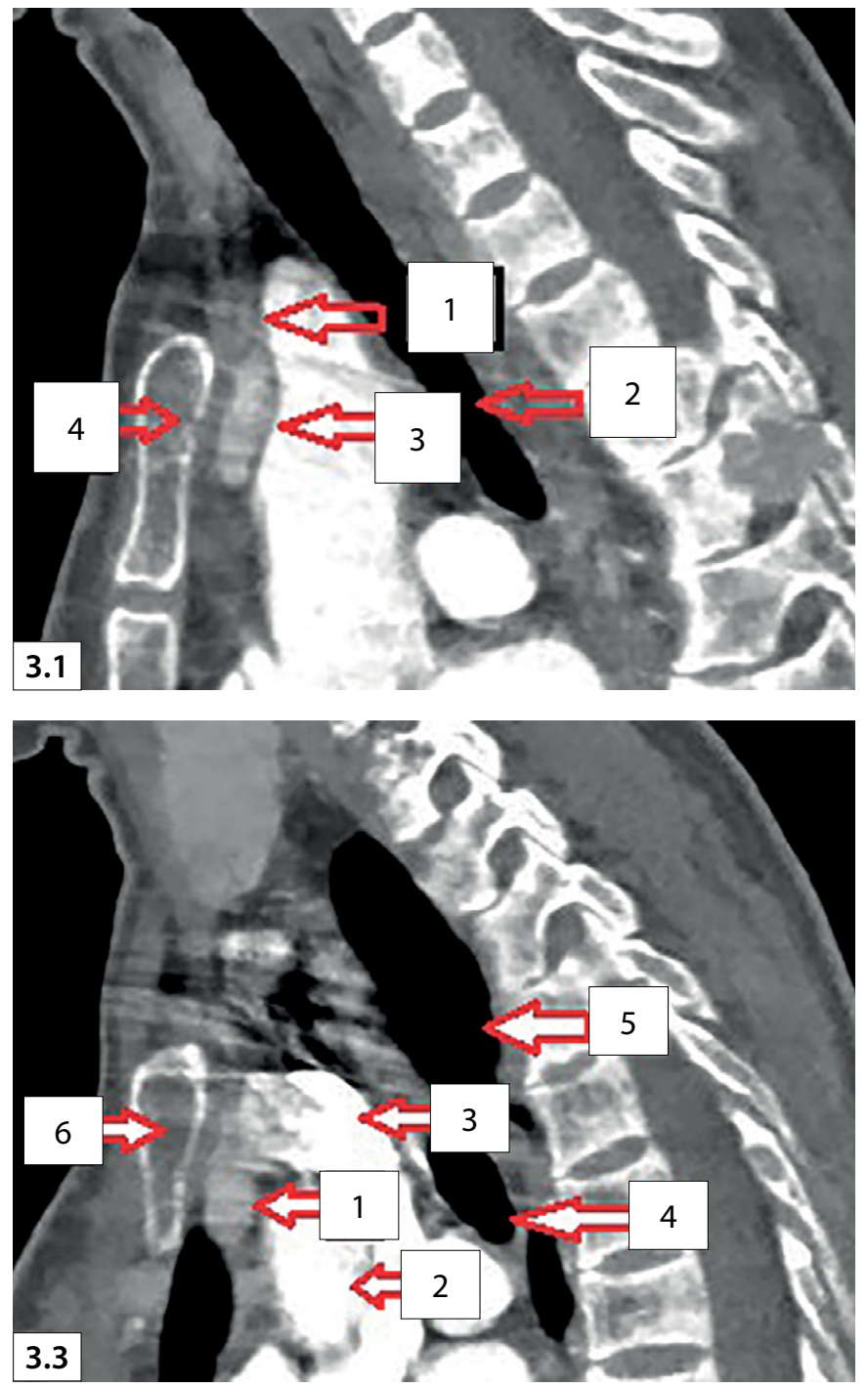

этих методов приводит к увеличению ДТ до 75\% [19]. При обнаружении атипично расположенных ОЩЖ ДТ данных методов визуализации существенно снижается: УзИ $36,7 \%$, сцинтиграфия - 30\%, МСКТ - 47,8\%, а сочетание методов - 63,3\% [19]. ОФЭКТ/КТ показывает наибольшую ДТ - 87-90\% по сравнению с традиционными методами визуализации ОЩЖ (сцинтиграфия - 59\%; УЗИ - 65\%) у пациентов с ВГПТ [20-21]. ДТ ОФЭКТ/КТ для обнаружения типично расположенных ОЩЖ - 89,5\%, а для атипично расположенных - 90,0\% [21]. Сочетание ОФЭКТ/КТ со сцинтиграфией позволяет увеличить ДТ до $72,12 \%$, а с УЗИ - до 79,39\% [22]. Считается, что сочетание ОФЭКТ/КТ с УЗИ - наилучший выбор для предоперационной визуализации ОЩЖ и снижения частоты персистенции/рецидива ВГПТ [23].

В настоящее время консенсуса в отношении объема оперативного вмешательства при ВГПт не достигнуто. Chen J. и соавт. (2017) сравнили эффективность субтотальной ПТЭ и тотальной ПТЭ с АТ. Авторы не обнаружили статистически значимых различий в отношении персистенции/рецидива ВГПТ $(p=0,45)$ и повторного оперативного
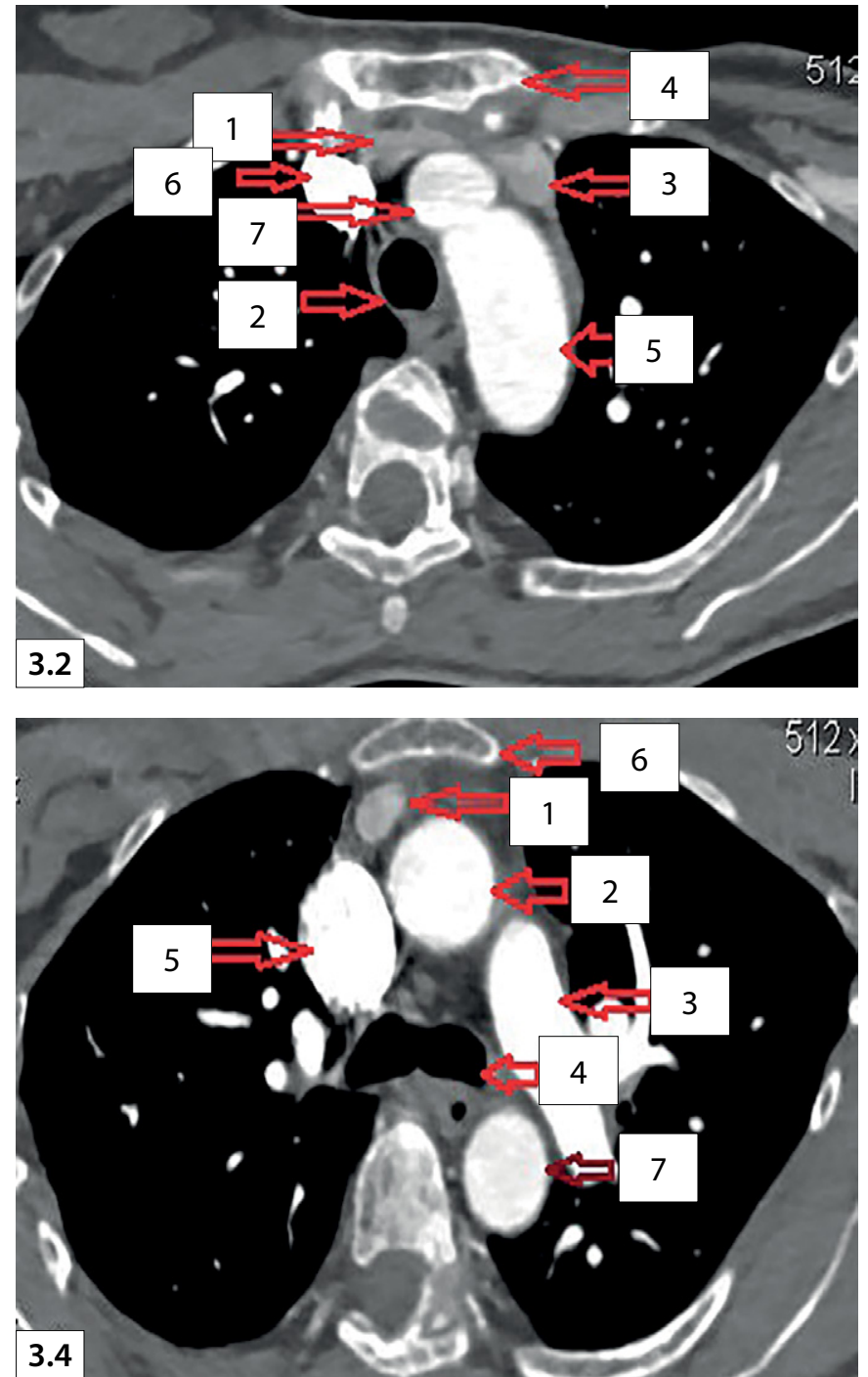

Рисунок 3. МСКТ-грамма. Мультиспиральная компьютерная томография шеи с ангиографией с внутривенным контрастированием.

Стрелками указаны анатомические структуры. 3.1 - сагиттальная проекция: 1 - левая нижняя Ощж; 2 - трахея; 3 - левая плечеголовная вена; 4 - рукоятка грудины. 3.2 - аксиальная проекция: 1 - левая плечеголовная вена; 2 - трахея; 3 - левая нижняя ОЩж; 4 - рукоятка грудины; 5,7 - дуга аорты; 6 - правая плечевая артерия. 3.3 - сагиттальная проекция: 1 - правая нижняя ОЩж; 2, 3 - дуга аорты; 4 - бифуркация трахеи; 5 - пищевод; 6 - рукоятка грудины; 3.4 - аксиальная проекция:1 - правая нижняя ОЩж; 2 - дуга аорты; 3 - брахиоцефальный ствол; 4 - бифуркация трахеи; 5 - правая подключичная артерия; 6 - рукоятка грудины; 


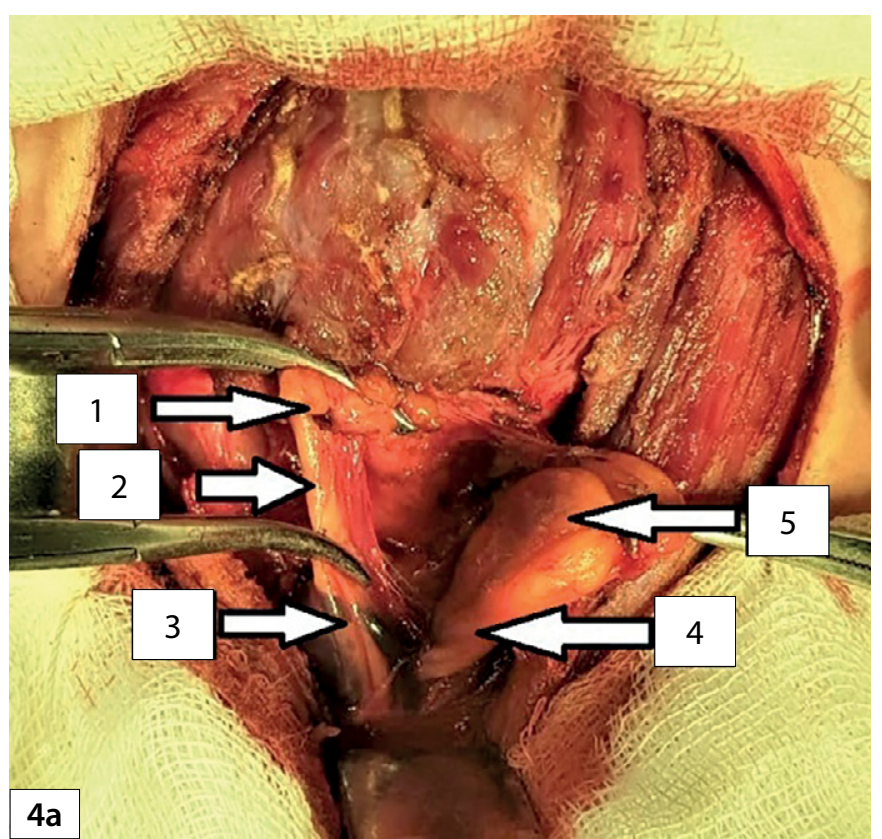

Рисунок 4а. Интраоперационная фотография.

Стрелками обозначены анатомические структуры. 1 - левая нижняя ОЩЖ; 2 - тиротимическая связка; 3 - левый верхний рог тимуса; 4 - правый верхний рог тимуса; 5 - правая нижняя ОЩЖ.

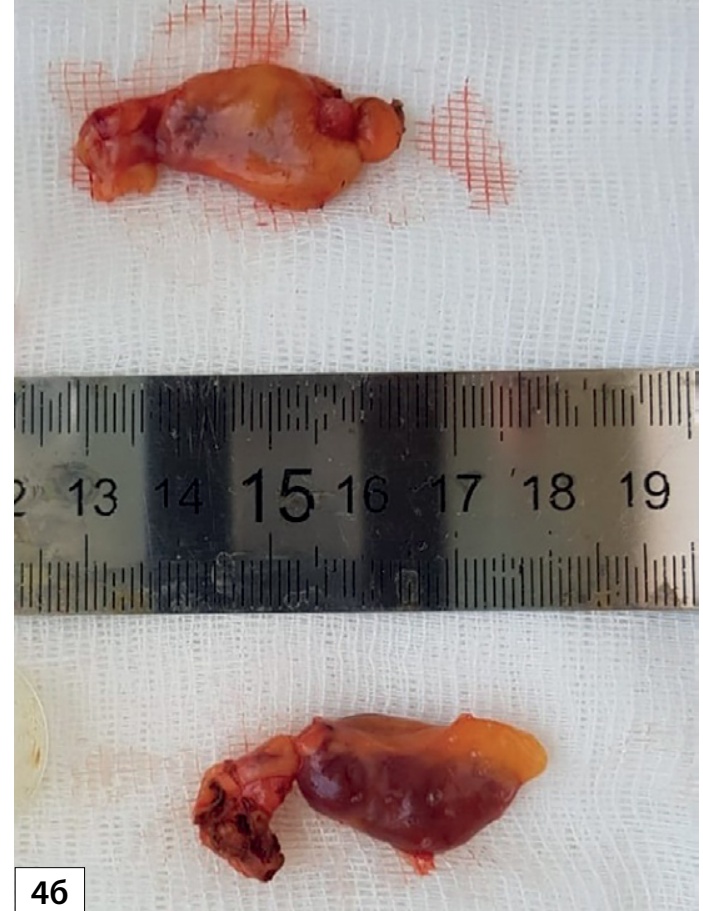

Рисунок 46. Макропрепараты.

Сверху — правая нижняя ОЩЖ, снизу — левая нижняя ОЩЖ.

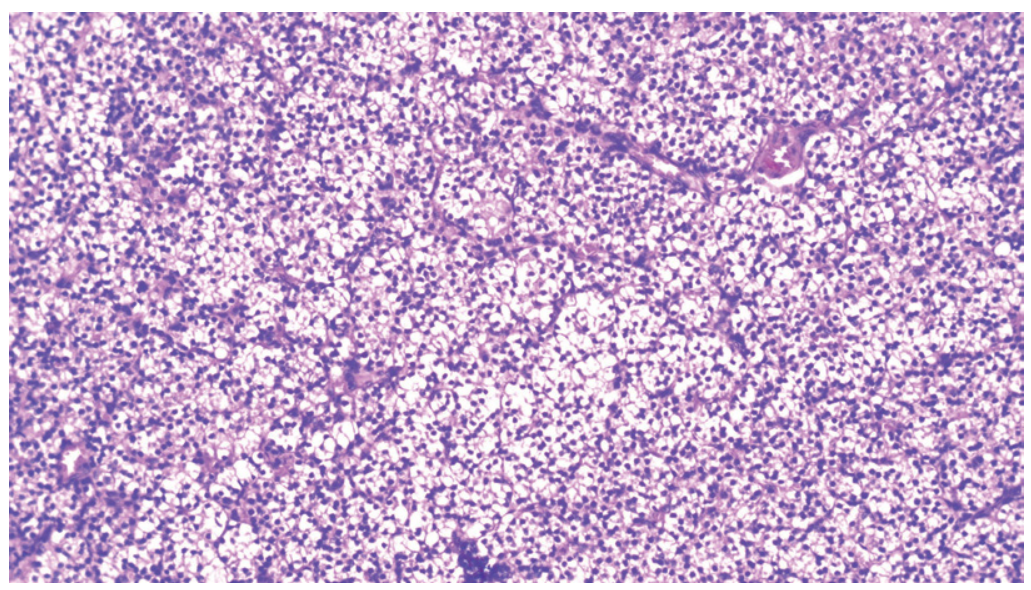

Рисунок 5. Микрофотография операционного материала. Окраска гематоксилином-эозином. Увеличение 10×0,25. Ткань нижней правой ОЩж. Все пространство препарата представлено картиной диффузной гиперплазии из главных темных клеток, без собственной капсулы и ободка неизмененной ткани. Аналогичной картиной представлен микропрепарат нижней левой ОЩЖ.

вмешательства $(p=0,35)$ [24]. Schlosser К. и соавт. (2016) оценили отдаленные результаты тотальной ПТЭ с АТ и без АТ, не выявив статистически значимых различий в отношении персистенции/рецидива ВГПТ [25].

Сохраняется дискуссия в отношении эффективности интраоперационного мониторинга ПТГ при ВГПТ. Данная методика является полезной для быстрой оценки эффективности операции по уровню снижения ПТГ после удаления ОЩж $[26,27]$. Одни авторы считают оптимальным снижение ПТГ от исходного уровня больше чем на 50-90\% [27, 28], а другие - дореференсных значений ПТГ [29]. Показана положительная роль интраоперационного мониторинга для быстрой диагностики добавочных или атипично расположенных ОЩЖ, которые не были выявлены в предоперационном периоде и не обнаружены во время выполнения ПТЭ [30].

В представленном клиническом наблюдении локализация ОЩЖ была установлена по данным УЗИ и сцинтиграфии (см. рис. 1) в рамках предоперационного обследования до первого оперативного вмешательства. Причину персистенции ВГПТ в данном случае мы видим в недостаточно полной оценке предоперационного обследования, недостаточном объеме оперативного вмешательства без ревизии рогов тимуса и неправильной интерпретации результатов интраоперационного мониторинга ПТГ во время первого оперативного вмешательства. Дообследование (повторное УЗИ, сцинтиграфия и МСКТ шеи с внутривенным усилением) (см. рис. 2, 3) позволило точно установить, что нижние ОЩЖ с обеих сторон располагаются в рогах тимуса. Поэтому во время оперативного вмешательства мы прицельно шли на ревизию рогов тимуса, что способствовало успеху оперативного вмешательства (рис. 4 а, б). Кроме того, подтверждением эффективности операции явилась положительная проба интраоперационного мониторинга ПТГ. 
В обсуждаемой истории болезни пациентка после операции принимала высокие дозы препаратов кальция и активных метаболитов витамина D. C одной стороны, это связано с развитием синдрома «голодных костей», с другой - послеоперационным гипопаратиреозом.

Синдром «голодных костей» наблюдается у $51,2-87,8 \%$ пациентов, которые перенесли ПТЭ по поводу ВГПТ [31-33]. В основе лежит резкое снижение резорбции костной ткани, активация остеобластов с формированием костной ткани. Проявляется длительной гипокальциемией с развитием клинических симптомов (парестезии и судорожная активность) [31-33]. У нашей пациентки описанный синдром обусловлен тяжелым поражением скелета в виде остеопороза (минимальный Z-показатель по данным остеоденситометрии составлял -3,8 в поясничном отделе позвоночника).

Гипопаратиреоз - заболевание, в основе которого лежит дефицит ПТГ, что приводит к гипокальциемии и в ряде случаев к гиперфосфатемии и гиперкальциурии. Среди всех случаев послеоперационный гипопаратиреоз составляет 75\% [34]. Транзиторный послеоперационный гипопаратиреоз развивается в 43,9\% после тотальной ПТЭ без АТ, 29\% - после тотальной ПТЭ с АТ и 45,5\% - после субтотальной ПТЭ [35]. Стойкий характер гипопаратиреоза возникает в 1\% после тотальной ПТЭ без АТ, 0,7-5,26\% - после тотальной ПТЭ с АТ и 4,5-8\% - после субтотальной ПтЭ [35-37].

Дефицит ПТГ является фактором развития адинамической болезни кости (АБК) [38]. В ее основе лежит дефект формирования и минерализации костного матрикса, проявляющийся метастатической кальцификацией и частыми переломами [39]. АБК является фактором риска сердечно-сосудистых осложнений за счет развития сосудистой кальцификации [40]. Јіа Х. и соавт. (2015) на основе метаанализа, включающего долгосрочные результаты после
ПТЭ по поводу ВГПТ на фоне ХБП у 931 пациента, заключают, что ни у одного из исследуемых пациентов не зарегистрировано наличие АБК и тяжелой гипокальциемии [41]. В настоящее время нет доказанного отрицательного влияния низкого уровня ПТГ и субклинической гипокальциемии в раннем и отдаленном послеоперационном периоде у больных на ЗПТ. Необходимы дальнейшие исследования по изучению долгосрочного влияния стойкого гипопаратиреоза у пациентов с терминальной стадией ХБП.

\section{ЗАКЛЮЧЕНИЕ}

С точки зрения профилактики персистенции и рецидивов ВГПТ оптимальным вариантом хирургического лечения является двусторонняя ревизия шеи, тотальная ПтЭ с удалением верхних рогов тимуса с использованием интраоперационного мониторинга ПтГ и последующей АТ ткани ОЩЖ. Необходима полная оценка результатов предоперационных методов визуализации при планировании оперативного вмешательства. Интраоперационный мониторинг ПтГ является эффективной методикой для оценки радикальности оперативного лечения.

\section{ДОПОЛНИТЕЛЬНАЯ ИНФОРМАЦИЯ}

Источник финансирования. Исследование и публикация статьи осуществлены на личные средства авторского коллектива.

Согласие пациента. Информированное согласие пациента на публикацию персональной медицинской информации в обезличенной форме было получено.

Конфликт интересов. Авторы декларируют отсутствие явных и потенциальных конфликтов интересов, связанных с публикацией настоящей статьи.

Участие авторов. Все авторы внесли значимый вклад в подготовку статьи, прочли и одобрили финальную версию до публикации.

\section{СПИСОК ЛИТЕРАТУРЫ | REFERENCES}

1. Дедов И.И., Мельниченко Г.А. Эндокринология. Клинические рекомендации. М.: ГЭОТАР-Медиа; 2017. [Dedov II, Mel'nichenko GA. Endokrinologiya. Klinicheskie rekomendatsii. Moscow: GEOTAR-Media; 2017. (In Russ.)].

2. Бикбов Б.Т., Томилина Н.А. Заместительная терапия больных с хронической почечной недостаточностью методами перитонеального диализа и трансплантации почки в Российской Федерации в 1998-2011 гг. // Нефрология и диализ. - 2014. T. 16. — №2. —C. 192-227. [Bikbov BT, Tomilina NA. Zamestitel'naya terapiya bol'nykh s khronicheskoi pochechnoi nedostatochnost'yu metodami peritoneal'nogo dializa i transplantatsii pochki v Rossiiskoi Federatsii v 1998-2011 gg. Nefrologiya i dializ. 2014;16(2):192-227. (In Russ.)].

3. Ассоциация нефрологов, Научное общество нефрологов России, Ермоленко В.М., Ветчинникова О.Н., Волгина Г.В., Гуревич К.Я., Томилина Н.А., Федосеев А.Н., Шутов Е.В., Андрусев А.М., Земченков А.Ю., Котенко О.Н., Михайлова Н.А., Ряснянский В.Ю. Шило В.Ю., Герасимчук Р.П. Минеральные и костные нарушения при хронической болезни почек. Начиональные рекомендации [Электронный ресурс]. 2015. Доступно по: http://www.nephro. ru/content/files/recomendations/ckdmbdNationalGuidelines. pdf [Association of Nephrology, Scientific Society of Nephrology of Russia, Ermolenko VM, Vetchinnikova ON, Volgina GV Gurevich KY, Tomilina NA, Fedoseev AN, Shutov EV, Andrusev AM, Zemchenkov AY, Kotenko ON, Mikhaylova NA, Ryasnyanskiy VY, Shilo VY, Gerasimchuk RP. 2015. Mineral and bone disorders in chronic kidney disease. National recommendations. Available from: http://www.nephro.ru/content/files/recomendations/ ckdmbdNationalGuidelines.pdf. (In Russ.)].

4. Bikbov B, Bieber B, Andrusev A, et al. Hemodialysis practice patterns in the Russia Dialysis Outcomes and Practice Patterns Study (DOPPS), with international comparisons. Hemodialysis/nt. 2017;21 (3):393-408. doi: https://doi.org/10.1111/hdi.12503

5. Герасимчук Р.П., Земченков А.Ю., Новокшонов К.Ю., и др. Влияние паратиреоидэктомии на динамику лабораторных показателей МКН ХБП и выживаемость пациентов, получающих заместительную почечную терапию диализом в Санкт-Петербурге // Нефрология и диализ. - 2016. - Т. 18. - №1. - С. 40-49. [Gerasimchuk RP, Zemchenkov AY, Novokshonov KY, et al. Influence of parathyroidectomy on the dynamics of laboratory indicators of MBD of CKD and survival of patients receiving renal replacement therapy in St. Petersburg. Nefrologiya i dializ. 2016;18(1):40-49. (In Russ.)].

6. Tsai W, Tsai W, Peng Y, et al. Short — and Long-Term Impact of Subtotal Parathyroidectomy on the Achievement of Bone and Mineral Parameters Recommended by Clinical Practice Guidelines in Dialysis Patients: A 12-Year Single-Center Experience. BloodPurif. 2013;36:116-121. doi: https://doi.org/10.1159/000353415

7. Егшатян Л.В., Мокрышева Н.Г., Рожинская Л.Я. Вторичный и третичный гиперпаратиреоз при хронической болезни почек // Ocmeonopoз и остеопатии. - 2017. - T. 20. №2. - C. 63-68. [Egshatyan LV, Mokrisheva NG, Rozhinskaya LY. Secondary and tertiary hyperparathyroidism in chronic kidney disease. Osteoporos Bone Dis. 2017;20(2):63-68. (In Russ.)]. doi: https://doi.org/10.14341/osteo2017263-68 
8. KDIGO 2017 Clinical Practice Guideline Update for the Diagnosis, Evaluation, Prevention, and Treatment of Chronic Kidney DiseaseMineral and Bone Disorder (CKD-MBD). Kidney Int Suppl. 2017;7(1):1-59. doi: https://doi.org/10.1016/j.kisu.2017.04.001

9. Величко А.В., Ярец Ю.И., Зыблев СЛ., и др. Влияние паратиреоидэктомии на показатели костного метаболизма у пациентов с вторичным гиперпаратиреозом на фоне хронической болезни почек // Новости хирургии. - 2016. - Т. 24. №1. - C. 32-39. [Velichko AV, Yarets Yl, Zyblev SL, et al. Effect of Parathyroidectomy on the Bone Metabolism Indicators in Patients with Secondary Hyperparathyroidism Against the Background of Chronic Renal Disease. Nov Khirurgii. 2016;24(1):32-39. (In Russ.)]. doi: https://doi.org/10.18484/2305-0047.2016.1.32

10. Rothmund M, Wagner PK, Schark C. Subtotal parathyroidectomy versus total parathyroidectomy and autotransplantation in secondary hyperparathyroidism: A randomized trial. World J Surg. 1991:15(6):745-750. doi: https://doi.org/10.1007/BF01665309

11. Schneider R, Slater EP, Karakas E, et al. Initial parathyroid surgery in 606 patients with renal hyperparathyroidism. World J Surg. 2012;36(2):318-326. doi: https://doi.org/10.1007/s00268-011-1392-0

12. Steffen L, Moffa G, Müller PC, Oertli D. Secondary hyperparathyroidism: recurrence after total parathyroidectomy with autotransplantation. Swiss Med Wkly. 2019;149:w20160. doi: https://doi.org/10.4414/smw.2019.20160

13. Sari $R$, Yabanoglu $H$, Hargura AS, et al. Outcomes of Total Parathyroidectomy with Autotransplantation versus Subtotal Parathyroidectomy Techniques for Secondary Hyperparathyroidism in Chronic Renal Failure. J Coll Physicians Surg Pak. 2020;30(1):18-22. doi: https://doi.org/10.29271/jcpsp.2020.01.18

14. Liu ME, Qiu NC, Zha SL, et al. To assess the effects of parathyroidectomy (TPTX versus TPTX+AT) for Secondary Hyperparathyroidism in chronic renal failure: A Systematic Review and Meta-Analysis. Int J Surg. 2017:44:353-362. doi: https://doi.org/10.1016/j.jisu.2017.06.029.

15. Abruzzo A, Gioviale MC, Damiano G, et al. Reoperation for persistent or recurrent secondary hyperparathyroidism. Acta Biomed. 2017;88(3):325-328. doi: https://doi.org/10.23750/abm.v88i3.4722

16. Konturek A, Barczyński M, Stopa M, NowakW. Subtotal parathyroidectomy for secondary renal hyperparathyroidism: a 20-year surgical outcome study. Langenbecks Arch Surg. 2016;401 (7):965-974. doi: https://doi.org/10.1007/s00423-016-1447-7

17. Sakman G., Parsak C.K., Balal M. Outcomes of Total Parathyroidectomy with Autotransplantationversus Subtotal Parathyroidectomy with Routine Addition of Thymectomy to both Groups: Single Center Experience of Secondary Hyperparathyroidism. Balkan Medical Journal. 2014;31 (1):77-82. doi: https://doi.org/10.5152/balkanmedj.2014.9544

18. Uludag M., Yetkin G., Citgez B. The role of cervical thymectomy in surgical treatment of secondary hyperparathyroidism. Bratis/LekListy. 2011;112(7):385-389

19. Hiramitsu T, Tomosugi T, Okada M, et al. Pre-operative Localisation of the Parathyroid Glands in Secondary Hyperparathyroidism: A Retrospective Cohort Study. Sci Rep. 2019;9(1):14634. doi: https://doi.org/10.1038/s41598-019-51265-y

20. Chen YH, Chen HT, Lee MC, et al. Preoperative F-18 fluorocholine $\mathrm{PET} / \mathrm{CT}$ for the detection of hyperfunctioning parathyroid glands in patients with secondary or tertiary hyperparathyroidism: comparison with Tc-99m sestamibi scan and neck ultrasound. Ann Nucl Med. 2020;34(8):527-537. doi: https://doi.org/10.1007/s12149-020-01479-2

21. Zhang R, Zhang Z, Huang P, et al. Diagnostic performance of ultrasonography, dual-phase $99 \mathrm{mTC}-\mathrm{MIBI}$ scintigraphy, early and delayed 99mTC-MIBI SPECT/CT in preoperative parathyroid gland localization in secondary hyperparathyroidism. BMC Med Imaging 2020;20(1):91. doi: https://doi.org/10.1186/s12880-020-00490-3

22. Jiang SQ, Yang T, Zou Q, et al. The role of $99 \mathrm{mTC}-M I B I S P E C T / C T$ in patients with secondary hyperparathyroidism: comparison with 99mTcMIBI planar scintigraphy and ultrasonography. BMC Med Imaging. 2020;20(1):115. doi: https://doi.org/10.1186/s12880-020-00517-9

23. Li X, Li J, LiY, et al. The role of preoperative ultrasound, contrastenhanced ultrasound, and 99mTc-MIBI scanning with single-photon emission computed tomography/X-ray computed tomography localization in refractory secondary hyperparathyroidism. Clin Hemorheol Microcirc. 2020;75(1):35-46. doi: https://doi.org/10.3233/CH-190723

24. Chen J, Jia X, Kong X, et al. Total parathyroidectomy with autotransplantation versus subtotal parathyroidectomy for renal hyperparathyroidism: A systematic review and meta-analysis. Nephrology (Carlton). 2017;22(5):388-396. doi: https://doi.org/10.1111/nep.12801
25. Schlosser K, Bartsch DK, Diener MK, et al. Total Parathyroidectomy With Routine Thymectomy and Autotransplantation Versus Total Parathyroidectomy Alone for Secondary Hyperparathyroidism: Results of a Nonconfirmatory Multicenter Prospective Randomized Controlled Pilot Trial. Ann Surg. 2016;264(5):745-753. doi: https://doi.org/10.1097/SLA.0000000000001875

26. Sakman G, Parsak CK, Balal M. Outcomes of Total Parathyroidectomy with Autotransplantationversus Subtotal Parathyroidectomy with Routine Addition of Thymectomy to both Groups: Single Center Experience of Secondary Hyperparathyroidism. Balkan Medical Journal. 2014;31(1):77-82. doi: https://doi.org/10.5152/balkanmedj.2014.9544

27. Weber T, Zeier M, Hinz U. Impact of intraoperative parathyroid hormone levels on surgical results in patients with renal hyperparathyroidism. World Journal of Surgery. 2005;29(9):1176-1179. doi: https://doi.org/10.1007/s00268-005-7805-1

28. Damiano G, Gioviale MC, Maione C. Comparison Between Rapid Intraoperative and Central Laboratory Parathormone Dosage in Kidney Transplant Candidates. Transplantation Proceedings. 2016;48(2):311-314. doi: https://doi.org/10.1016/j. transproceed.2016.02.003

29. Vulpio C, Bossola M, Di Stasio E. Intraoperative parathyroid hormone monitoring through central laboratory is accurate in renal secondary hyperparathyroidism. Clinical Biochemistry. 2016;49(7-8):538-543. doi: https://doi.org/10.1016/j.clinbiochem.2016.01.012

30. Ильичева Е.А., Булгатов Д.А., Жаркая А.В., и др. Опыт хирургического лечения вторичного гиперпаратиреоза с использованием интраоперационного мониторинга паратиреоидного гормона // Таврический медико-биологический вестник. - 2017. - Т. 20. №3. - C. 46-51. [llyicheva EA, Bulgatov DA, Zharkaya AV, et al. Experience of surgical treatment of secondary hyperparathyroidism with intra-operational monitoring of parathyroid hormone. Tavricheskiy mediko-biologicheskiy vestnik. 2017;20(3):46-51. (In Russ.)].

31. Ge Y, Yang G, Wang N, Zha X, et al. Bone metabolism markers and hungry bone syndrome after parathyroidectomy in dialysis patients with secondary hyperparathyroidism. Int Urol Nephrol. 2019;51 (8):1443-1449. doi: https://doi.org/10.1007/s11255-019-02217-y

32. Latus J, Roesel M, Fritz P, et al. Incidence of and risk factors for hungry bone syndrome in 84 patients with secondary hyperparathyroidism. Int J Nephrol Renovasc Dis. 2013;6:131-137. doi: https://doi.org/10.2147/IJNRD.S47179

33. Mittendorf EA, Merlino JI, McHenry CR. Post-parathyroidectomy hypocalcemia: incidence, risk factors, and management. Am Surg. 2004;70(2):114-119

34. Гребенникова Т.А., Белая Ж.Е., Мельниченко Г.А. Гипопаратиреоз: современное представление о заболевании и новые методы лечения // Эндокринная хирургия. - 2017. - Т. 11. №2. - C. 70-80. [Grebennikova TA, Belaya ZE, Melnichenko GA. Hypoparathyroidism: disease update and new methods of treatment. Endocrine Surgery. 2017;11 (2):70-80. (In Russ.)]. doi: https://doi.org/10.14341/serg2017270-80

35. Su A, Gong Y, Wu W, et al. Effect of autotransplantation of a parathyroid gland on hypoparathyroidism after total thyroidectomy. Endocr Connect. 2018;7(2):286-294. doi: https://doi.org/10.1530/EC-17-0313

36. Neagoe RM, Mureşan M, Voidăzan S, et al. Subtotal parathyroidectomy versus total parathyroidectomy with autotransplant in secondary hyperparathyroidism - a single-centre prospective cohort of 43 patients. Endokrynol Pol. 2016;67(2):202-209. doi: https://doi.org/10.5603/EP.a2016.0013

37. Veyrat M, Fessi H, Haymann JP, et al. Conservative three-quarter versus subtotal seven-eighths parathyroidectomy in secondary hyperparathyroidism. Eur Ann Otorhinolaryngol Head Neck Dis. 2019;136(2):63-68. doi: https://doi.org/10.1016/j.anorl.2018.09.003

38. Brandenburg VM, Floege J. Adynamic bone diseasebone and beyond. Clin Kidney J. 2008;1(3):135-147. doi: https://doi.org/10.1093/ndtplus/sfn040

39. Hruska KA, Teitelbaum SL. Renal Osteodystrophy. NEngl J Med. 1995;333(3):166-175. doi: https://doi.org/10.1056/NEJM199507203330307

40. London GM. Arterial Calcifications and Bone Histomorphometry in End-Stage Renal Disease. J Am Soc Nephrol. 2004;15(7):1943-1951. doi: https://doi.org/10.1097/01.ASN.0000129337.50739.48

41. Jia X, Wang R, Zhang C, et al. Long-Term Outcomes of Total Parathyroidectomy With or Without Autoimplantation for Hyperparathyroidism in Chronic Kidney Disease: A Meta-Analysis. Ther Apher Dial. 2015;19(5):477-485. doi: https://doi.org/10.1111/1744-9987.12310 


\section{ИНФОРМАЦИЯ ОБ АВТОРАХ [AUTHORS INFO]}

*Берсенев Глеб Александрович, аспирант [Gleb A. Bersenev]; ORCID: 0000-0002-6887-8325; SPIN:1467-8503; e-mail: glbersenev17@gmail.com

Ильичева Елена Алексеевна, д.М.н., професcop [Elena A. Ilyicheva, MD, PhD, Professor]; ORCID 0000-0002-2081-8665; SPIN:3624-4643; e-mail: lena_isi@mail.ru

Булгатов Дмитрий Александрович, м.н.c. [Dmitry A. Bulgatov]; ORCID: 0000-0002-2440-0813;

SPIN:1498-3741; e-mail: bbd-x@mail.ru

Махутов Валерий Николаевич, К.М.Н., зав. торакальным хирургическим отделением ГБУз ИОКБ [Valeriy N. Makhutov, MD, PhD]; ORCID:0000-0001-7318-7193; SPIN:7627-5484; e-mail: iokb@iokb.ru

\section{ИНФОРМАЦИЯ}

Рукопись получена: 15.12.2020. Одобрена к публикации: 30.04.2021.

\section{ЦИТИРОВАТЬ:}

Ильичева Е.А., Берсенев Г.А., Булгатов Д.А., Махутов В.Н. Персистенция вторичного гиперпаратиреоза после ранее проведенной паратиреоидэктомии у пациентки с хронической болезнью почек: клинический случай // Эндокринная хирургия. — 2020. — Т. 14. — №3. - C. 19-26. doi: https://doi.org/10.14341/serg12693

\section{TO CITE THIS ARTICLE:}

llyicheva EA, Bersenev GA, Bulgatov DA, Makhutov VN. Persistence of secondary hyperparathyroidism after parathyroidectomy in a patient with chronic kidney disease: a case report. Endocrine surgery. 2020;14(3): 19-26. doi: https://doi.org/10.14341/serg12693 